\title{
Efectividad Antimicrobiana del Colutorio de Matricaria recutita, en Funcionarios de la Facultad de Odontología de la Universidad del Desarrollo, Chile
}

\author{
Antimicrobial Effectiveness of Matricaria recutita Mouthwash in the \\ Dentistry Faculty Employees of Universidad del Desarrollo, Chile
}

Verónica Cárcamo O.; Patricio Oliva M. \& Patricio González C.

CÁRCAMO, O. V.; OLIVA, M. P. \& GONZÁLEZ, C. P. Efectividad antimicrobiana del colutorio de Matricaria recutita, en funcionarios de la Facultad de Odontología de la Universidad del Desarrollo, Chile. Int. J. Odontostomat., 5(2):179-184, 2011.

RESUMEN: El colutorio de manzanilla es un producto natural, no produce tinciones, alteraciones del sabor, no es toxico y puede ser utilizado por pacientes embarazadas, adultos mayores y niños. Se realizó un estudio experimental, con el objetivo de determinar la efectividad antimicrobial del colutorio en base a el extracto de Matricaria recutita I, tipo manzanilla primavera Puelche, el cual fue fabricado por la Dirección de Ciencias Básicas de la Facultad de Odontología de la Universidad del Desarrollo, Chile. Mediante un muestreo no probabilístico se reclutó 32 pacientes, funcionarios de la Facultad de Odontología. La metodología consignó la aplicación del Colutorio de Manzanilla (CM) en 5 pacientes (grupo experimental), Suero Fisiológico (SF) en 6 pacientes (control negativo) y Clorhexidina al 0,12\% (CX) en 7 pacientes (control positivo). Se tomó muestras de la mucosa del carrillo y de superficie vestibular del primer molar superior, previo a la aplicación del colutorio y en 7 intervalos de tiempo; se cultivó en condiciones de aerobiosis a $37^{\circ} \mathrm{C}$ por 48 horas. Luego se realizó el recuento bacteriano. Los resultados se analizaron mediante ANOVA para varianzas homogéneas y Kruskall-Wallis para varianzas heterogéneas. Se demostró que el recuento bacteriano no presenta diferencias significativas en los tiempos analizados para mucosa (CM pvalue: 0,2507 . CX pvalue: 0,1769 . SF pvalue: 0,9397$)$ y para diente (CM pvalue: 0,2540 . CX pvalue: 0,2859. SF pvalue: 0,3471), observándose que ningún resultado otorga un pvalue $<$ a 0,05 , en los 7 tiempos de aplicación. De esta manera se concluyó que la frecuencia de uso clínico del CM, presenta una mayor disminución de carga bacteriana cada 4 a 6 horas.

PALABRAS CLAVE: manzanilla, enjuague bucal, antibacteriano, mucosa oral.

\section{INTRODUCCIÓN}

El estudio determina la efectividad clínica del colutorio de extracto de Matricaria recutita I, tipo manzanilla primavera Puelche (colutorio de manzanilla), en adultos reclutados en la Facultad de Odontología de la Universidad del Desarrollo.

La Matricaria recutita I, es una planta de origen europeo, que crece también en lugares como África y América. Algunos de los componentes de la manzanilla son: azuleno, alfa bisabolol, ácido cafeico, ácido tánico, ácido clorogénico, umbelliferona, apigenina, herniarina, luteolina, ligeras cantidades de carotenos, vitamina C y alcohol sesquiterpético (Gispert Abreu, 1998). Se plantea que el azuleno es el principal responsable de las propiedades antiinflamatorias de la manzanilla, aunque el mecanismo de su eficacia no se ha aclarado (Fleischer, 1985). En cambio, al alfa bisabolol se le atribuye una acción antiséptica y antiinfecciosa (Reinhold, 1992). La manzanilla tiene un buen control de los microorganismos de la cavidad oral, también, disminuye los signos clínicos de la gingivitis, como inflamación y sangramiento de las encías. La gran mayoría de los pacientes cuidan de su salud bucal me- 
diante el uso de cepillo, pasta y seda dental. Siendo este tipo de higiene, por lo general, insuficiente, ya que no se eliminan en su totalidad la gran cantidad de bacterias presentes en el medio (Bernimoulin, 2003). Se ha comprobado estadísticamente que los pacientes que utilizan removedores de placa bacteriana, versus aquellos que solo utilizan cepillado convencional, presentan un mejor control de la placa en un $75 \%$, evidenciándose una disminución de los niveles de enfermedad gingival (Grippaudo, 1995). El colutorio de manzanilla gracias a su composición natural, no presenta efectos secundarios ni contraindicaciones, como alguno de los otros colutorios existentes en el mercado; así ocurre con la clorhexidina que produce tinciones dentales después de 15 días de uso prolongado (Calsina-Gomis \& Serrano-Granger, 2005).

\section{MATERIAL Y MÉTODO}

Este estudio se realizó con el personal de la Facultad de Odontología de Universidad del Desarrollo (adulto sano). Mediante muestreo no probabilístico se seleccionó 32 sujetos que cumplieron con los criterios de inclusión y de exclusión. Este estudio incluyó a todos aquellos sujetos de sexo femenino, con 20 dientes en boca como mínimo (cuatro molares y dos incisivos centrales en boca como mínimo). Se excluyó a todos los sujetos que presentaban consumo de antiinflamatorios o que se encontraban utilizando cualquier enjuague bucal. Todos los procedimientos realizados estuvieron de acuerdo con Declaración de Helsinki de 1975, enmendada en el año 2005. Se usó el colutorio de manzanilla (CM) como intervención y como grupos controles se utilizó clorhexidina al 0,12\% (CX), que correspondió al control positivo y suero fisiológico (SF), que correspondió al control negativo.

Para recolectar los datos, se dividió el estudio en 8 tiempos, siendo el tiempo 0 la completación de la ficha clínica, que incluyó: datos del paciente, examen dentario y aplicación del índice gingival de Löe Silness (Löe, 1967), este índice se aplicará también al llegar a la última muestra, para comprobar el estado de los tejidos gingivales; Tiempo 1: muestra bacteriológica de mucosa y encía inmediatamente después de realizada una profilaxis bucal completa; Tiempo 2: muestra bacteriológica de mucosa y encía después de utilizado el enjuague (CM, CX o SF); Tiempo 3: muestra bacteriológica de mucosa y encía transcurrida media hora post enjuague; Tiempo 4: muestra bacteriológica de mucosa y encía transcurrida 1 hora post enjuague;
Tiempo 5: muestra bacteriológica de mucosa y encía transcurridas 2 horas post enjuague; Tiempo 6: muestra bacteriológica de mucosa y encía transcurridas 4 horas post enjuague; Tiempo 7: muestra bacteriológica de mucosa y encía transcurridas 8 horas post enjuague. Para finalizar, la aplicación del índice gingival y de Löe Silness.

La metodología consignó la aplicación del CM en 5 pacientes, suero fisiológico en 6 pacientes y clorhexidina al $0,12 \%$ en 7 pacientes. Estos cultivos bacteriológicos se tomaron con conos de papel, previamente esterilizados en autoclave (SHINVA $®$ TMQ.R 3250), tanto del tercio cervical vestibular de la estructura dentaria (pieza 1.6 o primer molar superior derecho), como de la mucosa del carrillo, ambas muestras fueron tomadas durante 30 segundos, lográndose la saturación del cono de papel. Las muestras fueron conservadas en un medio de cultivo y transporte, que correspondió a caldo trioglicolato (BBL® Thioglycollate, Medium), en donde se utilizó 1,7 mL en tubo Eppendorf, luego fueron incubadas en el horno de cultivo a una temperatura constante de $37^{\circ} \mathrm{C}$ por 48 horas, esperando crecimiento y desarrollo bacteriano.

Este estudio se dividió en dos tiempos metodológicos, una metodología de toma de muestras y otra metodología de cultivo microbiológico.

La metodología de toma de muestras, comenzó con una profilaxis bucal completa con un scaler (Biosonic® US100R. Ultrasonic Scaler). Luego se aplicó el índice gingival de Löe Silness. Después se procedió a la toma de muestra con el cono de papel en mucosa y diente, que corresponde al tiempo 1. Luego se le pidió al paciente que se enjuague durante 1 minuto con $5 \mathrm{~mL}$ de CM, SF o CX y se procede a la toma de muestra, tiempo 2, inmediatamente después de la aplicación del enjuague. A la media hora post enjuague se procedió a la toma de muestra, tiempo 3 . A 1 hora post enjuague se procedió a la toma de muestra, tiempo 4. A las 2 horas post enjuague se procedió a la toma de muestra, tiempo 5 . A las 4 horas post enjuague se procedió a la toma de muestra, tiempo 6 . A las 8 horas post enjuague se procedió a la toma de muestra, tiempo 7 .

Finalmente se aplicó el índice gingival de Löe Silness, para comprobar posibles alteraciones o efectos secundarios a corto plazo en la cavidad bucal, después de la utilización de los enjuagues.

La metodología de cultivo microbiológico, correspondió a todos los procedimientos desde que se toma- 
ron las muestras desde la cavidad bucal, hasta el conteo de UFC en las placas de Agar BHI. Las muestras obtenidas se colocaron en tubos Eppendorf que contenían en su interior caldo trioglicolato como medio de transporte, estos tubos fueron llevados al horno a $37^{\circ} \mathrm{C}$ durante dos días, esperando el crecimiento bacteriano, que se evidencia con un aspecto turbio del líquido del tubo Eppendorf. Transcurridos los dos días, las muestras fueron sembradas en placas de agar BHI (Agar Brain Heart Infusion. HIMEDIA® M211), este agar es el medio de cultivo ideal para bacterias aerobias y anaerobias presentes en la cavidad bucal, siendo enriquecido con levadura (BBL $®$ Yeast Extract) y sangre para facilitar el crecimiento bacteriano. Las placas fueron sembradas e incubadas durante 48 horas a $37^{\circ} \mathrm{C}$, evidenciándose crecimiento o desarrollo bacteriano. Posteriormente se procedió a contar las UFC utilizando un contador electrónico de colonias (CP600 Plus, Phoenix®).

El instrumento de recolección de datos correspondió a la ficha clínica, que incluyó los datos personales del paciente, anamnesis médica remota y próxima, examen dental e Índice Gingival de Löe Silness. Para el análisis inferencial se ocupó la prueba de análisis de varianzas ANOVA, bajo el supuesto de varianzas homogéneas medidos a través del Test de Bartlett's y también se ocupó el Test de Kruskall-Wallis si no se cumpliera el supuesto de normalidad. Todo el análisis inferencial se utilizó con un nivel de significación menor al $5 \%$.

\section{RESULTADOS}

Se realizó análisis estadístico para el CM, la CX 0,12\% CX y el SF, respecto al sitio de aplicación, mucosa y diente, y al crecimiento de las UFC en los 7 tiempos de muestras.

Se decidió utilizar el test de ANOVA para varianzas homogéneas y el test de Kruskall-Wallis en caso de existir varianzas heterogéneas. Mediante el Test de ANOVA y de Kruskall-Wallis se demostró que el recuento bacteriano no presenta diferencias significativas en los tiempos analizados para mucosa (CM pvalue $=0,2507$. CX pvalue $=0,1769$. SF pvalue $=0,9397$ ) y para diente $(\mathrm{CM}$ pvalue $=0,2540$. $\mathrm{CX}$ pvalue $=0,2859$. $\mathrm{SF}$ pvalue $=$ 0,3471), como se observa en Tablas I, III, IV, V y VII.

Tabla I. Análisis de varianzas para colutorio de manzanilla en mucosa con test de ANOVA.

\begin{tabular}{cccccc}
\hline Fuente & SS & df & MS & F & Prob > f \\
\hline Entre grupos & $2,4188 \mathrm{e}+09$ & 5 & 483767836 & 1,45 & $\mathbf{0 , 2 5 0 7}$ \\
Dentro de grupos & $6,3183 \mathrm{e}+09$ & 19 & 332539968 & & \\
\hline Total & $8,7371 \mathrm{e}+09$ & 24 & 364045774 & & \\
\hline
\end{tabular}

Tabla II. Valores para clorhexidina $0,12 \%$ en Mucosa mediante el test no paramétrico de Kruskall-Wallis.

\begin{tabular}{ccc}
\hline Tiempo & Obs & Sum Rangos \\
\hline 1 & 6 & 88,00 \\
2 & 5 & 79,00 \\
3 & 5 & 69,50 \\
4 & 5 & 54,00 \\
5 & 5 & 86,50 \\
7 & 6 & 151,00 \\
chi-cuadrado $=$ & 7,645 con & 5 d.f. \\
probabilidad= & $\mathbf{0 , 1 7 6 9}$ & \\
\hline
\end{tabular}

Tabla III. Análisis de varianzas para suero fisiológico en mucosa con test de ANOVA.

\begin{tabular}{cccccc}
\hline Fuente & SS & df & MS & F & Prob > f \\
\hline Entre grupos & 883404710 & 5 & 176680942 & 0,24 & $\mathbf{0 , 9 3 9 7}$ \\
Dentro de grupos & $1,4683 e+10$ & 20 & 734152284 & & \\
Total & $1,5566 e+10$ & 25 & 622658015 & & \\
\hline
\end{tabular}


Tabla IV. Análisis de varianzas para colutorio de manzanilla en diente con test de ANOVA.

\begin{tabular}{cccccc}
\hline Fuente & SS & df & MS & F & Prob > f \\
\hline Entre grupos & $3,8892 e+09$ & 5 & 777836687 & 1,44 & $\mathbf{0 , 2 5 4 0}$ \\
Dentro de grupos & $1,0232 e+10$ & 19 & 538516962 & & \\
Total & $1,4121 e+10$ & 24 & 588375238 & & \\
\hline
\end{tabular}

Tabla V. Valores para clorhexidina $0,12 \%$ en diente mediante el test no paramétrico de Kruskall-Wallis.

\begin{tabular}{ccc|}
\hline Tiempo & Obs & Sum Rangos \\
\hline 1 & 6 & 92,50 \\
2 & 5 & 83,00 \\
3 & 5 & 76,00 \\
4 & 5 & 67,00 \\
5 & 5 & 55,50 \\
7 & 6 & 122,00 \\
chi-cuadrado $=$ & 6,214 with & 5 d.f. \\
\hline probabilidad $=$ & $\mathbf{0 , 2 8 5 9}$ & \\
\hline
\end{tabular}

Tabla VI. Valores para Suero Fisiológico en Diente mediante el test no paramétrico de Kruskall-Wallis.

\begin{tabular}{ccc|}
\hline Tiempo & Obs & Sum Rangos \\
\hline 1 & 6 & 107,00 \\
2 & 5 & 97,00 \\
3 & 5 & 44,00 \\
4 & 5 & 82,00 \\
5 & 6 & 126,00 \\
6 & 5 & 72,00 \\
chi-cuadrado $=$ & 5,600 with & 5 d.f. \\
probabilidad $=$ & $\mathbf{0 , 3 4 7 1}$ & \\
\hline
\end{tabular}

\section{DISCUSIÓN}

Los resultados de este estudio muestran que el mejor momento para que el paciente se enjuague con el CM es cada 4 a 6 horas (Fig. 1). Este horario coincide con el horario de comidas de los pacientes, que en una dieta saludable debe ser cada 4 horas, por lo tanto el paciente después de comer realizará su rutina de higiene oral y se enjuagará con el CM. Respecto al control positivo, que correspondió al enjuague de CX al 0,12\%, éste presenta un mayor tiempo de detención en el crecimiento bacteriológico, hasta aproximadamente 4 horas post enjuague (Fig. 2), como ya se nombró anteriormente, este tipo de enjuague bucal tiene efectos secundarios indeseables (tinciones dentarias y de restauraciones, sabor metálico, alteraciones del gusto) frente a un uso prolongado (mayor a 15 días). Respecto al control negativo, que correspondió al SF, éste disminuye la carga bacteriana principalmente por el efecto de arrastre que se produce cuando el paciente realiza un enjuague (Fig. 3).

Bajo el microscopio electrónico (Olympus CX21), a un aumento de 1000x, se pudo evidenciar que el tipo de bacterias presentes en las placas de agar correspondió a Streptococcus y Staphylococcus
Gram + (Fig. 5.1 y Fig. 5.2); éstas bacterias son de tipo aerobias o anaerobias facultativas, es decir que viven en un medio donde hay oxígeno. Éstas bacterias están fuertemente relacionadas con la presencia de gingivitis, por lo tanto se puede deducir que el CM tiene importantes propiedades antibacterianas, específicamente sobre estos dos tipos de bacterias, y llega a los sitios donde un cepillado convencional no lo hace, como es en las superficies interdentarias, evidenciándose en los resultados del estudio de Gispert et al., en donde se usó una pasta dental en base a manzanilla y se observó una disminución considerable en el nivel de Streptococcus. A nivel epidemiológico las patologías orales de mayor prevalencia en nuestro país son: caries dentales, enfermedades gingivales y periodontales y anomalías dento maxilares. No obstante, existe una prevalencia de gingivitis en un 2,6\% de los niños de 2 años; $6,2 \%$ a los 4 años; $55,09 \%$ a los 6 años; $66,9 \%$ a los 12 años; $75 \%$ en adultos (MINSAL, 2008). Los datos demuestran que el nivel de enfermedades gingivales y periodontales tienen relación directa con la edad, por lo tanto es de vital importancia promover hábitos saludables de alimentación e higiene bucal desde temprana edad, mejorando así el nivel de salud oral para toda la familia. 


\section{Colutorio de Manzanilla}

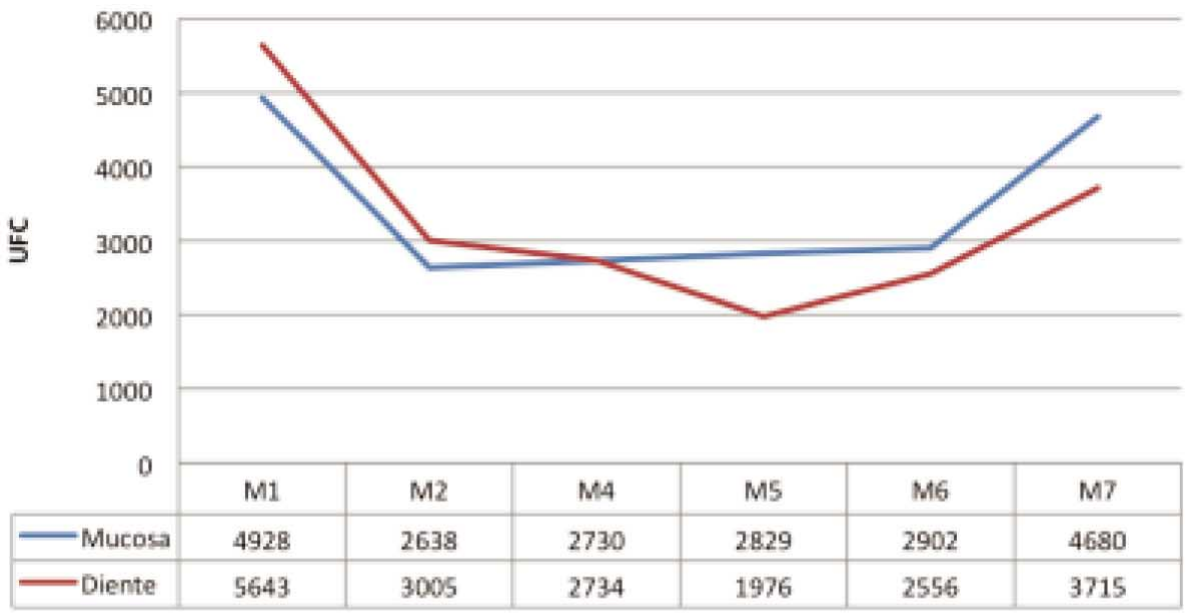

Fig. 1. Valores descriptivos para colutorio de manzanilla en mucosa y diente.

\section{Clorhexidina}

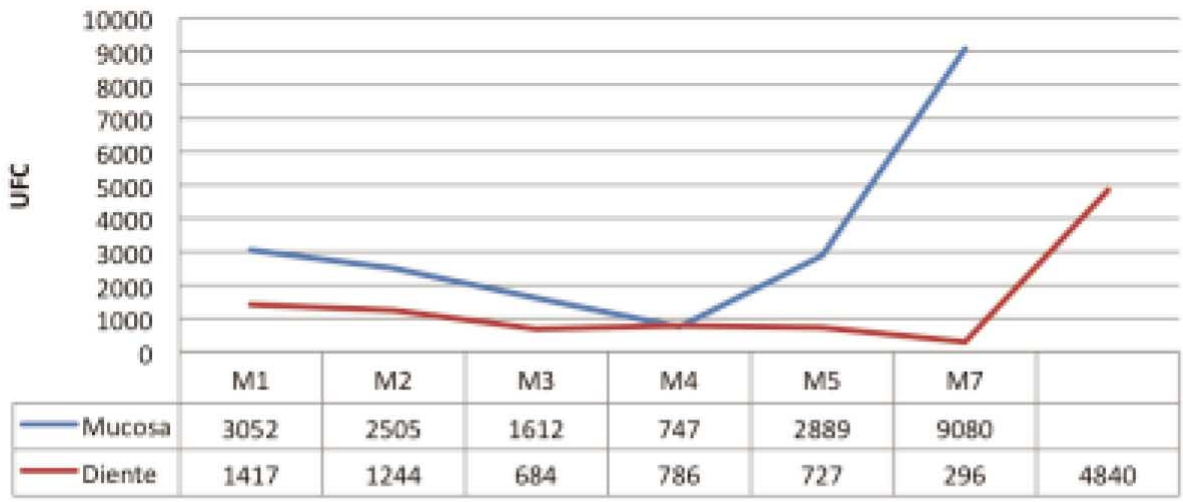

Fig. 2. Valores descriptivos para Clorhexidina $0,12 \%$ en mucosa y diente.

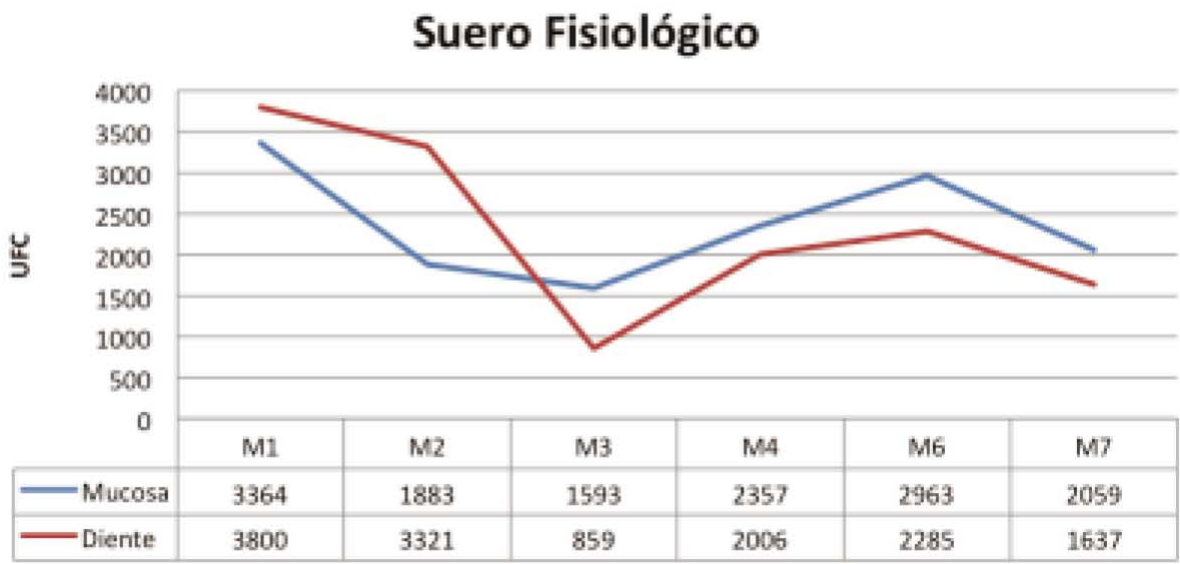

Fig. 3. Valores descriptivos para suero fisiológico en mucosa y diente. 


\section{CONCLUSIONES}

La utilización optima del colutorio de extracto de Matricaria recutita I, tipo manzanilla primavera Puelche, es cada 4 a 6 horas, evidenciándose una mejor disminución de la carga bacteriana.

Se evidencian diferencias en el recuento bacteriano de cada una de los enjuagues utilizados. A pesar de ello, no existe diferencia significativa en el recuento bacteriano en los 7 tiempos de muestro en mucosa para CM (pvalue $=0,2507), \mathrm{CX} 0,12 \%$ (pvalue $=0,1769$ ) y SF (pvalue $=0,9397$ ); $y$ en diente para CM (pvalue $=0,2540$ ), Clorhexidina $0,12 \%$ (pvalue= 0,2859 ) y SF (pvalue $=0,3471$ ). No obstante, el uso del CM sería importante como coadyudante en la higiene oral de los pacientes, lo que motiva a continuar investigando los efectos beneficiosos del colutorio, frente a patologías más prevalentes de la cavidad oral.

CÁRCAMO, O. V.; OLIVA, M. P. \& GONZÁLEZ, C. P. Antimicrobial effectiveness of Matricaria recutita mouthwash in the dentistry faculty employees of the Universidad del Desarrollo. Int. J. Odontostomat., 5(2):179-184, 2011.

ABSTRACT: Chamomile mouthwash is a natural product, does not cause stains, taste disturbances, is non-toxic and can be used by pregnant patients, the elderly and children. An experimental study was performed with the aim of determining the antimicrobial effectiveness of mouthwash produced from Matricaria recutita extract I, Puelche Manzanilla Primavera Type, which was produced by the Department of Basic Sciences, of the Dentistry Faculty of the Universidad del Desarrollo. Thirty two patients were recruited using a non-probability sampling, employees from the School of Dentistry. The methodology of work was defined by the application of chamomile mouthwash (CM) in 5 patients (experimental group), saline (SF) in 6 patients (negative control) and $0.12 \%$ chlorhexidine (CX) in 7 patients (positive control). Samples were taken from the cheek mucosa and upper first molar bucal surface, prior to the application of chamomille mouthwash and 7 time intervals. The samples were grown under aerobic conditions at $37^{\circ} \mathrm{C}$ for 48 hours. This was followed by bacterial count. The results, like homogeneous variance, were analyzed by ANOVA and Kruskall-Wallis to heterogeneous variances. It was shown that the bacterial count did not differ significantly with the time analyzed for mucosa (CM pvalue: 0.2507 . CX pvalue: 0.1769, Pvalue SF: 0.9387 and tooth (CM pvalue <0.2540). CX pvalue: 0.2859. pvalue SF: 0.3471) showing that no result gives a pvalue $<0.05$, within 7 days of application. Thus it was concluded that the frequency of clinical use of chamomile mouthwash, presents a greater reduction in bacterial load every 4 to 6 hours.

KEY WORDS: Chamomile, Mouthwash, Antibacterial, oral mucosa.

\section{REFERENCIAS BIBLIOGRÁFICAS}

Bernimoulin, J. P. Conceptos recientes sobre formación de placa. J. Clin. Periodontol., 30:7-9, 2003.

Calsina-Gomis, G. \& Serrano-Granger, J. ¿Existen realmente diferencias clínicas entre las distintas concentraciones de clorhexidina? Comparación de colutorios. RCOE, 10(4):457-64, 2005.

Fleischer, A. M. Plants Extract: To accelerate healing and reduce inflammation. Cosmetic Toilet, 100:14753, 1985.

Gispert Abreu, E.; Cantillo Estrada, E.; Rivero López, A. \& Oramas Rodríguez, B. Crema dental con manzanilla, efecto estomatológico. Rev. Cubana Estomatol., 35(3):107-11, 1998.

Grippaudo, G. Estudio clínico sobre la eficacia de un nuevo tipo de removedor de la placa bacteriana. $J$. Periontol., 1(2):995-6, 1995.
Löe, $\mathrm{H}$. The gingival index, the plaque index and the retention index system. J. Periodontol., 386:Suppl. 610-6, 1967.

MINSAL. Diagnóstico de Situación de Salud Bucal (2008, Septiembre). Disponible en: http:// www.redsalud.gov.cl/archivos/salud_bucal/ perfilepidemiologico.pdf

Reinhold C. Producción de un extracto de manzanilla con cualidades curativas. Sol 92100387 . F. Sol 23.01.91, NP 04962 30, F.Pub. 11.01.92. RFA. 1992.

Dirección para correspondencia:

Verónica Cárcamo $\mathrm{O}$.

Facultad de Odontología

Universidad del Desarrollo

Concepción

CHILE

Recibido : 20-07-2011

Aceptado: 16-08-2011 